\title{
ANÁlISE DO CICLO DE VIDA NO AQUECIMENTO DOMÉSTICO DE ÁGUA
}

\author{
Vinícius Velanes Giffoni ${ }^{1}$; Diego Lima Medeiros²; Eduardo Cohim² \\ ${ }^{1}$ Programa de Pós-Graduação em Engenharia Civil e Ambiental - UEFS; Feira de \\ Santana/Bahia; vvelanes@hotmail.com \\ 2 Programa de Pós-Graduação em Engenharia Industrial - UFBA; Salvador/Bahia \\ ${ }^{3}$ Programa de Pós-Graduação em Engenharia Civil e Ambiental - UEFS; Feira de \\ Santana/Bahia
}

Resumo: O objetivo do trabalho foi a análise energética e da pegada de carbono do equipamento hídrico doméstico mais relevante no consumo de energia, o chuveiro elétrico. Para a elaboração da Análise do Ciclo de Vida (ACV), foi elaborado um inventário alimentado com dados como tempo médio de banho, volume de água gasto num banho, tempo de vida útil de um chuveiro, entre outros. Também foram avaliados os impactos energéticos e de mudanças climáticas através da demanda de energia acumulada (CED) e potencial de aquecimento global (GWP). Com os resultados da pesquisa, pretende-se complementar o valor do impacto energético no consumo de um metro cúbico de água dentro da residência, valor este que já foi sugerido em 3,51 $\mathrm{kWh} / \mathrm{m}^{3}$ de água efetivamente usada.

Palavras-Chave: Indicadores; Avaliação do Ciclo de Vida (ACV); eficiência energética.

\section{LIFE CYCLE ANALYSIS IN WATER DOMESTIC HEATING}

\begin{abstract}
The objective of this work was the energy and carbon footprint analysis of the most relevant energy consumption domestic water equipment, the electric shower. For the elaboration of Life Cycle Analysis (LCA), an inventory was elaborated fed with data such as average bath time, volume of water spent in a bath, shelf life of a shower, among others. Energy and climate change impacts were also assessed through cumulative energy demand (CED) and global warming potential (GWP). With the results of the research, it is intended to complement the value of the energy impact in the consumption of one cubic meter of water inside the residence, which has already been suggested in $3.51 \mathrm{kWh} / \mathrm{m}^{3}$ of water actually used.
\end{abstract}

Keywords: Indicators; Life Cycle Assessment (LCA); energy efficiency. 


\section{INTRODUÇÃO}

A escassez da água potável vem se tornando um problema global. A economia nos setores de abastecimento e consumo de água e energia é uma condição indispensável para o desenvolvimento econômico e social da sociedade contemporânea. Nesse aspecto, o setor de saneamento merece atenção especial no que diz respeito à possibilidade de economia desses dois insumos por meio da busca da eficiência no dimensionamento e uso de certos equipamentos domésticos. Segundo pesquisas da Agência Americana de Proteção do Meio Ambiente [1], os usuários de água e esgoto consomem cerca de 30 a $60 \%$ das contas de energia elétrica de uma cidade.

Além disso, o crescente consumo de energia no mundo, estimulado mais recentemente pelo desenvolvimento acelerado dos países emergentes, vem aportando impactos significativos na economia em escala global. A escalada do consumo repercute no custo da energia, impactando de maneira generalizada o preço de diversos produtos essenciais para a vida moderna [2].

Dentro desse quadro de escassez hídrica, as deficiências do setor de abastecimento de aliadas ao consumo desordenado por parte da população, além de contribuir para o alto consumo de energia, aumentam as emissões de gases de efeito estufa (GEE) resultantes da geração de energia. A energia consumida para distribuir a água potável corresponde a cerca de $7 \%$ do consumo energético mundial. Isto sem incluir a energia consumida dentro das residências e tratamento das águas residuais [3].

Com o crescimento da população dos grandes centros aliado ao aumento da demanda de água e energia, a interdependência entre esses dois recursos vem ficando mais evidente. De acordo com estudos, nos serviços urbanos de abastecimento de água, esgotos e saneamento, a energia é o principal componente do custo operacional [4]. O processo de distribuição de água e tratamento de águas residuais demanda um intenso uso de energia. Além disso, até a própria fabricação das redes de tubos em larga escala são dispendiosas energeticamente.

No Brasil, $3 \%$ do consumo de eletricidade é devido ao setor de abastecimento de água e tratamento de esgotos [2]. O consumo de eletricidade no país, em 2012, foi de aproximadamente 463 mil GWh, dos quais $2 \%$ da eletricidade anual foi utilizada para o setor de água [5]. Nesse mesmo ano, foram divulgados dados de uma pesquisa afirmando que $12 \%$ da energia ofertada no Brasil destinava-se exclusivamente para fins residenciais [6].

O uso racional da água engloba, portanto, a minimização dos desperdícios (por perda ou uso excessivo) e a maximização da eficiência do uso, sendo que, essa maximização se dá pela utilização de uma menor quantidade de água para realização das atividades, porém sem comprometer sua qualidade. Esse objetivo pode ser atingido tanto em função de características dos equipamentos quanto em função do nível de conscientização do usuário [7].

Ainda decorrente à preocupação por formas de mitigar os impactos ambientais causados pelo aumento da demanda dos recursos hídricos e desperdícios pelo 
abastecimento e uso dessa água, medidas de gestão dos recursos hídricos devem ser associadas à gestão ambiental. Os indicadores ambientais são amplamente utilizados como instrumento de apoio à decisão, ao quantificarem os resultados de ações de governo e auxiliarem o processo de avaliação da gestão [8].

O uso de indicadores de eficiência energética permite monitorar a eficiência energética do progresso e da economia; avaliar o impacto de políticas e programas voltados para eficiência energética, inclusive justificando a manutenção ou interrupção de ações; planejar ações futuras, incluindo programas de pesquisa e desenvolvimento (P\&D); alimentar modelos de projeções de modo a prever a demanda de energia futura; possibilitar comparações entre setores e países [9].

Tal abordagem permite que o controle da gestão dos sistemas de abastecimento e uso de água promova resultados mais eficientes e confiáveis a fim de colaborar com as tomadas de decisões dos governos e população que visem a diminuição dos problemas sociais e ambientais relacionados à escassez hídrica.

Uma das ferramentas mais utilizadas na gestão ambiental é a Análise de Ciclo de Vida (ACV), cujo conceito é definido como um processo de avaliação das implicações ambientais do produto, processo ou serviço, através da identificação e da quantificação da energia, materiais e emissões ambientais, avaliação do impacto ambiental e identificação de oportunidades para melhorias ambientais [10].

Um estudo de ACV tem sua metodologia baseada nas seguintes etapas: objetivo e escopo, análise do inventário, avaliação de impactos e interpretação dos resultados. Estas etapas são inter-relacionadas pois, desta forma, o problema é analisado e solucionado de maneiro holística [11,12].

Diversos equipamentos domésticos consomem energia no uso de água em residências. Entre estes, destaca-se o aquecimento de água em chuveiros elétricos, a gás ou termossolares, que, segundo pesquisa divulgada [6], contam com um índice de penetração de $71 \%$ nas residências, e são responsáveis por $18 \%$ do gasto energético total da residência. A demanda energética do uso de água na residência deve, portanto, considerar a parcela destes equipamentos.

Dados abordam o uso de chuveiros nas residências, onde afirmam que $73 \%$ dos domicílios brasileiros contam com o chuveiro com aquecedor elétrico e este pode chegar a ser responsável por $25 \%$ da energia de toda a residência [13].

Desta forma, com a finalidade de contribuir para a redução da parcela dos impactos ambientais domésticos, o objetivo deste trabalho é elaborar a Análise do Ciclo de Vida (ACV) da fabricação e operação dos aquecedores de água para o uso doméstico. Aplicando a ACV, serão quantificados os insumos e avaliados os impactos energéticos e climáticos gerados pelo uso destes aparelhos na residência.

\section{METODOLOGIA (ARIAL 12)}

Foi adotada a pesquisa exploratória por meio de dados disponíveis na literatura. Foi usada a base de dados de inventário ecoinvent 3.2 e o software Simapro 8 para avaliação dos impactos. Os métodos de potencial de aquecimento global (GWP) para 
emissão de gases de efeito estufa [14] e demanda de energia primária acumulada (CED) para energia [17]. Foram utilizados dados da base do Instituto Nacional de Metrologia, Normalização e Qualidade Industrial [15], International Energy Agency [16] Empresa de pesquisas energéticas [6] para definição do consumo energético dos equipamentos domésticos, assim como dados do Instituto Brasileiro de Geografia e Estatística [18] para aspectos como número de moradores por residência e consumo.

- Foram considerados os seguintes aparelhos domésticos de aquecimento de água: chuveiro elétrico, chuveiro a gás e chuveiro a energia termossolar. $O$ número de habitantes por residência foi retirado do Censo [18] para a cidade de Feira de Santana e corresponde a $3,47 \mathrm{hab} / \mathrm{res}$.

- O volume de água gasto no banho foi calculado com base na vazão média dos chuveiros disponibilizada em catálogos técnicos como 4,5 L/min e no tempo médio de banho, que, para a cidade de Feira de Santana é de 7,3 $\min [19]$.

Foi considerado 2 banhos / hab / dia e um tempo de vida útil de 5 anos para os chuveiros.

\section{RESULTADOS E DISCUSSÃO}

Foram feitos comparativos para os três tipos de chuveiro, utilizando os métodos de potencial de aquecimento global (GWP) do [14] para emissão de gases de efeito estufa e demanda de energia primária acumulada (CED). A Figura 1 apresenta 0 comparativo pelo método IPCC para os três tipos de chuveiro estudados.

Figura 1. Impacto climático comparativo por habitante por dia

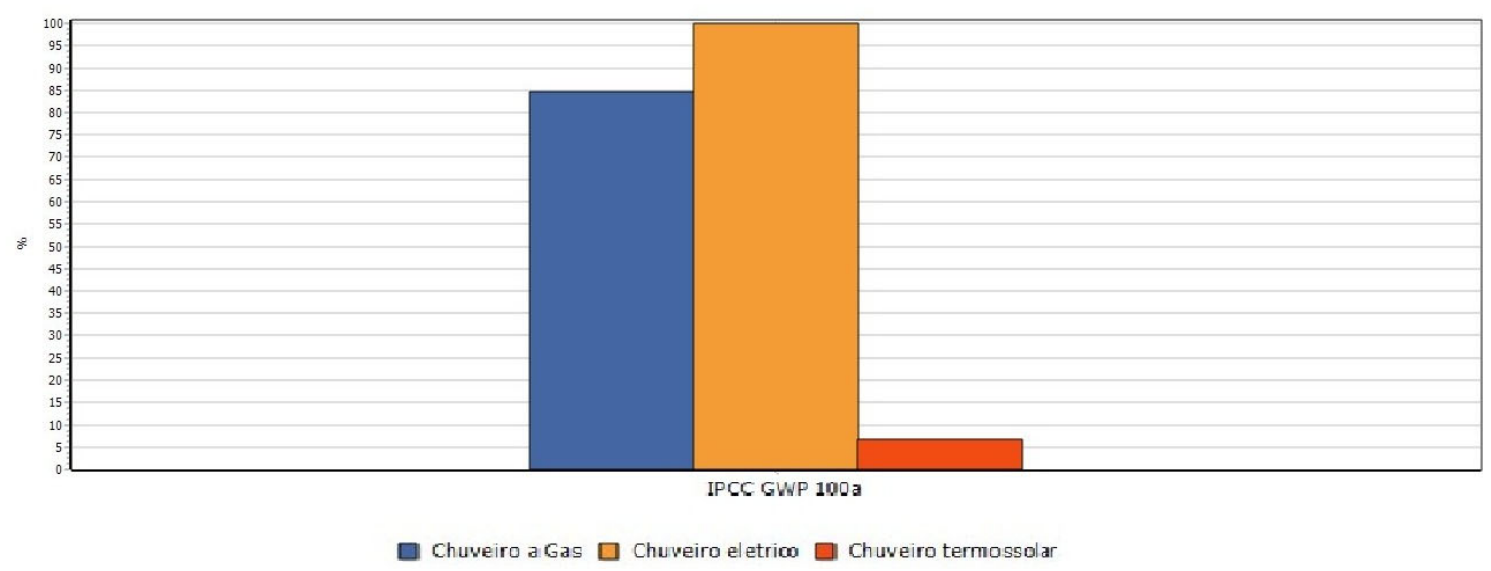

A coluna do centro representa o impacto climático causado pelo uso do chuveiro elétrico a cada um habitante por dia. Este é o tipo de chuveiro que emite a maior quantidade de $\mathrm{CO}_{2} \mathrm{eq} / \mathrm{hab} / \mathrm{dia}$, correspondente a 1,59 kg de $\mathrm{CO}_{2} \mathrm{eq}$. $\mathrm{O}$ 
chuveiro com aquecimento termossolar, por sua vez, tem o menor impacto climático dos três, emitindo apenas $0,106 \mathrm{~kg}$ de $\mathrm{CO}_{2} \mathrm{eq} / \mathrm{hab} / \mathrm{dia}$, correspondente a $13 \%$ do impacto do chuveiro elétrico. Uma das justificativas para isto é o fato deste tipo de equipamento não necessitar de energia elétrica para seu funcionamento

Figura 2. Impacto energético comparativo por habitante por dia

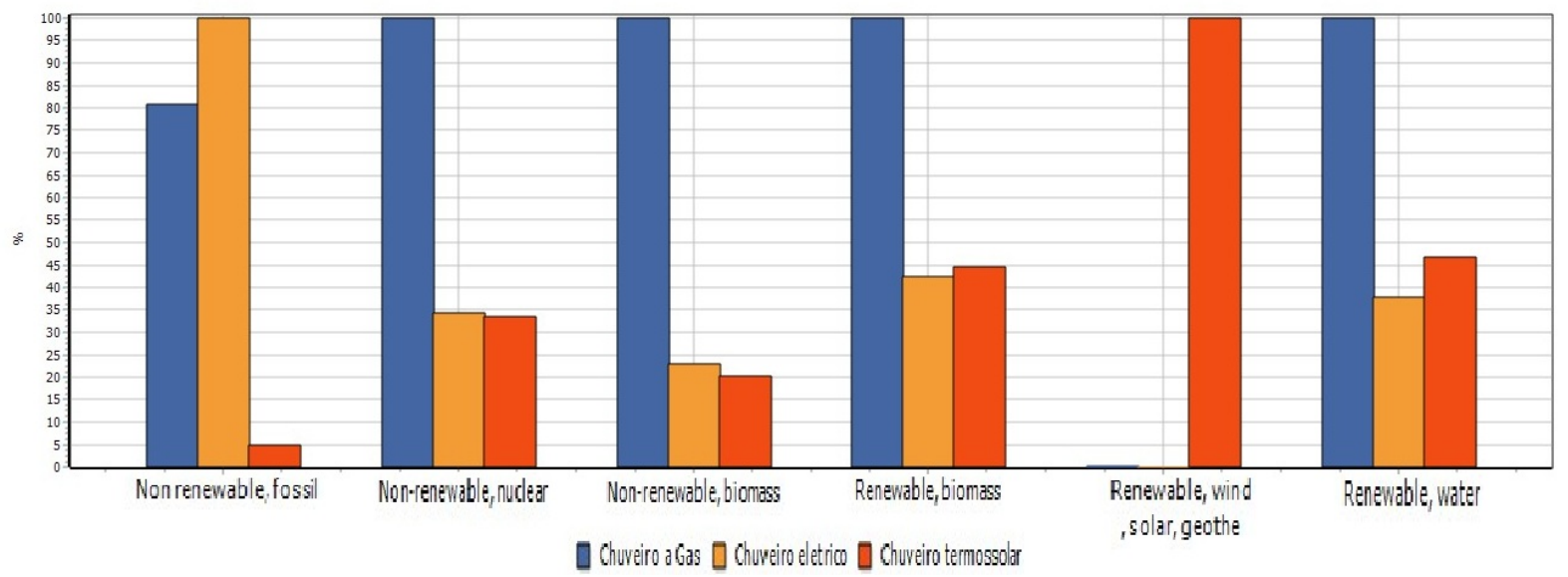

A figura 2 apresenta os impactos gerados pelos três tipos de chuveiro com cinco tipos recursos, são eles: fóssil, nuclear, biomassa, solar e água.

Com exceção do uso da energia solar, o chuveiro termossolar também foi o menos impactante em todos os aspectos, se destacando principalmente no uso dos recursos não-renováveis, a exemplo dos combustíveis fósseis.

O chuveiro a gás se mostrou mais impactante na maioria dos recursos, como nuclear, as biomassas e água. Entretanto, quando feita a análise unificada do ciclo de vida de cada tipo de chuveiro, o impacto energético do aquecedor a energia elétrica foi de 26,2 MJ / hab / dia, o que corresponde a 7,28 kWh (Figura 3), seguido dos aquecedores a gás e termossolar, com 6,06 kWh e 4,86 kWh, respectivamente.

A figura 4 detalha graficamente o impacto climático gerado pelo chuveiro elétrico em $\mathrm{CO}_{2} \mathrm{eq} / \mathrm{hab} / \mathrm{dia}$.

Figura 3. Impacto energético do chuveiro elétrico do habitante por dia. 


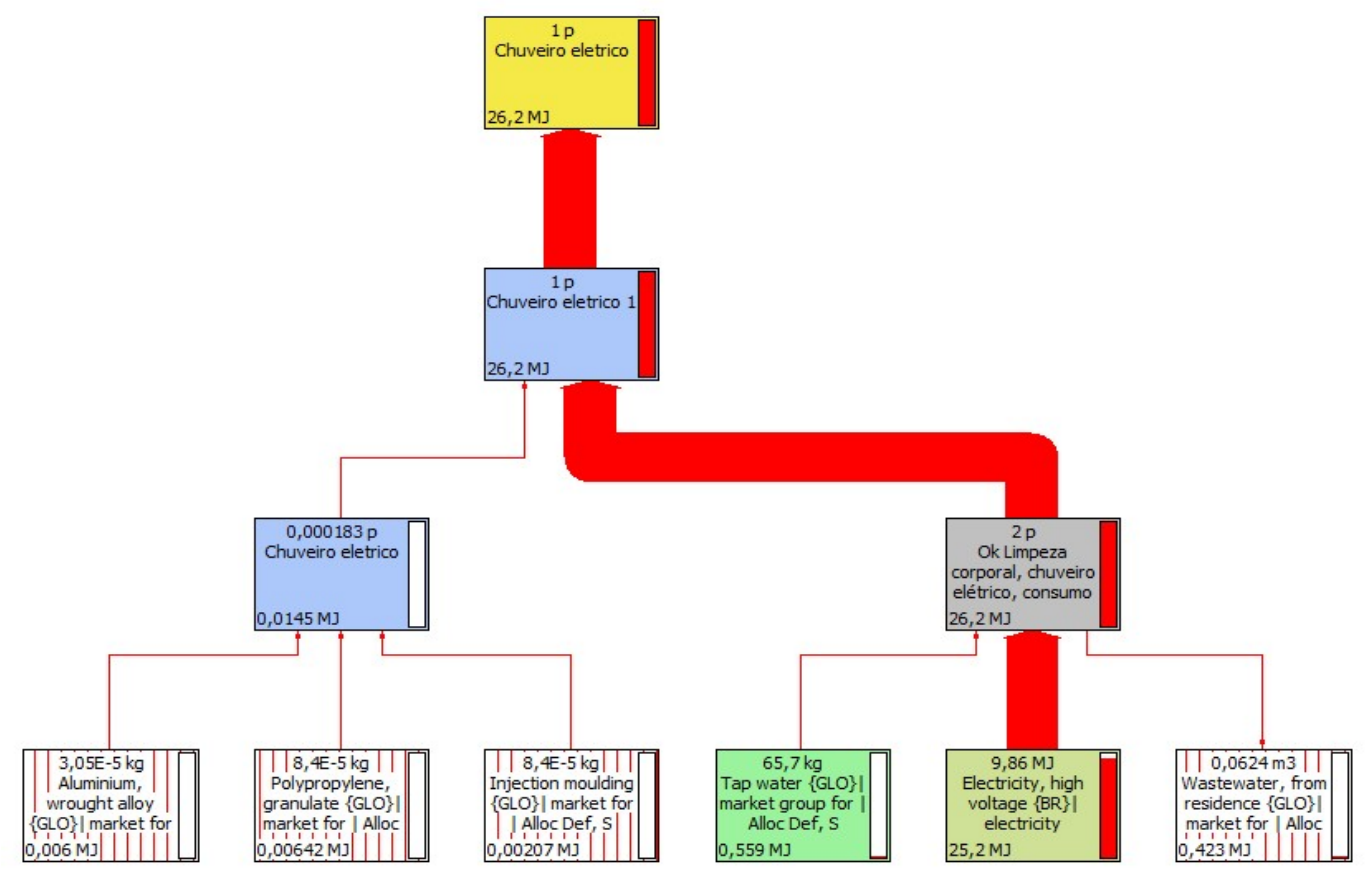

Como fica demonstrado no gráfico, a maior parcela do impacto provém do uso do chuveiro ao longo da sua vida útil, deixando impacto gerado pela sua montagem desprezível. Os três tipos de chuveiros seguem essa mesma configuração, diferindo apenas na energia utilizada e nos materiais de montagem.

Observa-se, portanto, a vantagem energética do uso de chuveiros a gás e termossolares em relação ao elétrico no consumo de energia. Por outro lado, o custo para instalação e manutenção desses aparelhos deve ser considerado.

Figura 4. Impacto climático do chuveiro elétrico do habitante por dia 


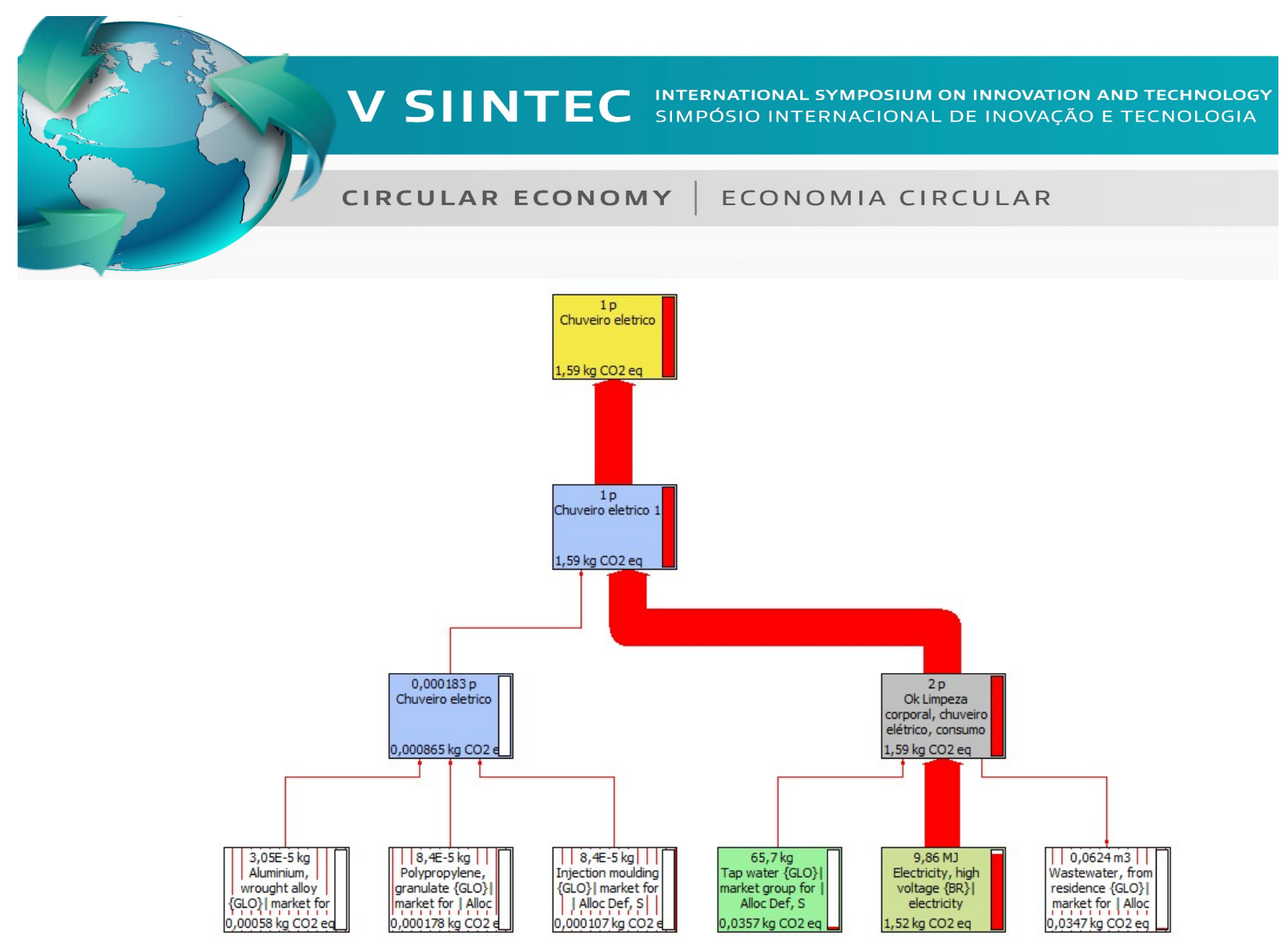

\section{CONCLUSÃO (ARIAL 12)}

A escolha do aparelho com o menor consumo de energia aliada ao consumo consciente por parte dos usuários são as ferramentas necessárias para diminuir os impactos ambientais causados pelo uso desses aparelhos. Conclui-se, portanto, que a energia embutida no uso de um chuveiro elétrico (incluindo sua fabricação) numa residência com 3,47 habitantes por dia por é de $25,3 \mathrm{kWh}$. Este valor, se comparado ao gasto energético para tratamento e abastecimento de $1 \mathrm{~m}^{3}$ de água, sugerida por [20] em 3,51 kWh, é relevante, e o estudo de maneiras que mitiguem estes danos são plenamente justificáveis.

Entre as principais soluções para a diminuição do gasto energético em residências, a mais efetiva é justamente o consumo dos usuários, pois, de acordo com a ACV dos chuveiros elétricos, reduzindo-se em 3 min o tempo de banho, o consumo energético pode cair em até $41 \%$.

Outro cenário possível é a utilização de tecnologias alternativas, a exemplo dos chuveiros que utilizam mais de um tipo de energia, chamados mistos, onde seu princípio de funcionamento é utilizar o aquecimento com energia elétrica durante a fase de ativação da energia termossolar, chegando assim, a um meio termo capaz de otimizar o gasto energético na residência.

\section{REFERÊNCIAS (ARIAL 12)}

${ }^{11}$ ASSOCIAÇÃO BRASILEIRA DE NORMAS TÉCNICAS - ABNT. NBR ISO 14040, de 2009 - Gestão ambiental - Avaliação do ciclo de vida - Princípios e estrutura. Rio de Janeiro. 2009a. 
${ }^{12}$ ASSOCIAÇÃO BRASILEIRA DE NORMAS TÉCNICAS - ABNT. NBR ISO 14044, de 2009 - Avaliação do Ciclo de Vida: Requisitos e Orientações. Rio de Janeiro: 2009b.

${ }^{5}$ BRASIL. MCTI. Segunda Comunicação Nacional do Brasil à Convenção-Quadro das Nações Unidas sobre Mudança do Clima. Brasília: Brasília: Secretaria de Política e Programas de Pesquisa e Desenvolvimento - SEPED, 2010. (Período: 1990 - 2005).

${ }^{6}$ CONSUMO DE ENERGIA NO BRASIL: Análises Setoriais. Rio de Janeiro: Empresa de Pesquisa Energética (EPE), 2014.

1 ENSURING A SUSTAINABLE FUTURE: AN ENERGY MANAGEMENT GUIDEBOOK FOR WASTEWATER AND WATER UTILITIES. Washington: U.S. Environmental Protection Agency (EPA), 2008.

20 GUANAIS, Ana Luiza da Silva Rezende. AVALIAÇÃo ENERGÉtICA E DAS EMISSÕES DE GASES DE EFEITO ESTUFA DO SISTEMA INTEGRADO DE ABASTECIMENTO DE ÁGUA DE FEIRA DE SANTANA. 2015. 118 f. Dissertação (Mestrado) - Curso de Mestrado em Engenharia Civil e Ambiental, Universidade Estadual de Feira de Santana, Feira de Santana, 2015.

18 IBGE - Instituto Brasileiro de Geografia e Estatística. Disponível em:<http://www.ibge.gov.br/home/>.Acesso em 28 out. 2018.

15 INMETRO - Instituto Nacional de Metrologia, Normalização e Qualidade Industrial. Eficiência Energética-Programa Brasileiro de Etiquetagem. Disponível em <http://www.inmetro.gov.br/qualidade/eficiencia.asp> Acesso em 22 out. 2018.

17 JUNGBLUTH, N., FRISCHKNECHT R (2007) Cumulative energy demand.Implementation of life cycle impact assessment methods. Ecoinvent report $\mathrm{n}^{\circ}$. 3, v2.0.Swiss Centre for Life Cycle Inventories.

${ }^{16}$ MIND THE GAP: Quantifying Principal-Agent Problems in Energy Efficiency. Paris: Internacional Energy Agency (IEA), 2007.

${ }^{8}$ MIRANDA, G. M.; BRAGA, R. Indicadores de desempenho na gestão dos recursos hídricos nos municípios das bacias hidrográficas dos rios Piracicaba, Capivari e Jundiaí. GEONORDESTE, Ano XXII, n.2. Aracajú, 2011.

9 ODYSSEE. Energy Efficiency Indicators in Europe. Disponível em: <http://www.odyssee-indicators.org/2011> Acesso em: 19 nov. 2018.

${ }^{13}$ PROCEL - Programa Nacional de Conservação de Energia Elétrica. Disponível em <https://www.eletrobras.com/elb/data/Pages/LUMIS0389BBA8PTBRIE.htm>. Acesso em 04 nov. 2018. 
${ }^{2}$ PROSAB (Programa de Pesquisa em Saneamento Básico) Caderno5.Ano2009. Uso racional de água e energia: Conservação de água e energia em sistemas prediais e públicos de abastecimento de água.

${ }^{19}$ SANTOS, J. F. R. Percepção dos professores da rede pública sobre usos e fontes alternativas de água em Feira de Santana-BA. 2016. 235p. Dissertação (Mestrado) - Universidade Estadual de Feira de Santana, Feira de Santana, 2016.

7 SILVA, G. S. Programa permanente de uso racional da água em Campi Universitários: o programa de uso racional da água da Universidade de São Paulo. 2004. 482p. Dissertação (Mestrado) - Escola Politécnica da Universidade de São Paulo, São Paulo, 2004.

4 THINKING ABOUT WATER DIFFERENTLY: managing water-food-energy nexus. Mandaluyong City: Asian Development Bank (ADB), 2013.

${ }^{3}$ TRIPATHI, M. Life-Cycle Energy and Emissions for Municipal Water and Wastewater Services:

CaseStudiesofTreatmentPlantsinUS.AnnArbor.CenterforSustainable Systems, University of Michigan - U.S. 2007.

${ }^{10}$ UNEP - United Nations Environment Programme. Guidelines for social life cycle assessment of products. France, 2009. 